\title{
El cuerpo en la lingüística cognitiva. La metáfora conceptual y el embodiment
}

\author{
Recibido: 15 de noviembre de 2016 \\ Aceptado: 20 de julio 2017 \\ Publicado: 27 de noviembre de 2017
}

\author{
Daniela Soledad Gonzalez \\ dsgonzalez@conicet.gov.ar \\ Universidad Nacional de Cuyo (Argentina)
}

\begin{abstract}
Resumen: El presente trabajo da cuenta del modo en el que la lingüística cognitiva aborda el tema del cuerpo en relación con el lenguaje, entendido este como un correlato del pensamiento. Uno de los ejes de abordaje del lenguaje que caracteriza a este enfoque teórico es el de la metáfora conceptual, noción postulada por Lakoff y Johnson (2001). Los autores conciben la metáfora como un fenómeno del pensamiento que se refleja en el lenguaje, que está omnipresente en la vida cotidiana y que permite la estructuración de la experiencia. La experiencia a la que hacen referencia es, fundamentalmente, corporal. La influencia de la experiencia corporal en la conceptualización recibe el nombre de embodiment o embodied mind (Lakoff, 1987: 12).
\end{abstract}

Palabras clave: Lingüística cognitiva, metáfora, embodiment.

\begin{abstract}
The present work approaches the way in which Cognitive Linguistics addresses the issue of the body in relation to language, which is understood as a correlate of thought. One of the axes of language addressing that characterizes this theoretical approach is the notion of conceptual metaphor postulated by Lakoff and Johnson (2001). These authors conceive metaphor as a phenomenon of thought that is reflected in language, is omnipresent in everyday life and allows the structuring of experience. The experience to which they refer is essentially corporal. The influence of body experience in conceptualizing is called embodiment or embodied mind (Lakoff, 1987: 12).
\end{abstract}

Key words: Cognitive Linguistics, Metaphor, Embodiment. 


\section{Introducción: el enfoque cognitivo-funcional de los fenómenos lingüísticos}

En la actualidad, un enfoque de los fenómenos del lenguaje se va consolidando: la lingüística cognitiva. Esta perspectiva de estudio sostiene que toda emisión implica una conceptualización de la experiencia que se busca comunicar, desde la elección de las desinencias flexivas hasta la estructura de la oración.

Una noción importante en los estudios cognitivo-funcionales del lenguaje es la de dominio cognitivo. Para Lakoff (1987), un dominio cognitivo es un "modelo cognitivo idealizado": un conjunto coherente de conocimientos de naturaleza enciclopédica, a veces muy simplificados e incluso equivocados ${ }^{1}$.

Algunos dominios cognitivos pueden ser el espacio, las acciones transitivas, los procesos y otros tipos de representaciones de la experiencia en las que confluyen diversos elementos significativos como el dominio de los utensilios de cocina, el de los tratamientos médicos, entre otros. La noción de dominio conceptual se aplica en un campo de estudio importante y desarrollado de la lingüística cognitiva: la metáfora conceptual y la metonimia (Lakoff y Johnson, 2001), que se explican a continuación, con el fin de explicar el modo en que pensamos y su relación con el cuerpo.

\section{La metáfora conceptual}

Lakoff y Johnson (2001) son autores ineludibles en el estudio de la metáfora como operación conceptual. Presentaron una concepción novedosa de la metáfora, afirmando que se trataba de un fenómeno del pensamiento que se reflejaba en el lenguaje, que estaba omnipresente en la vida cotidiana y que permitía la estructuración de la experiencia (Lakoff y Johnson, 2001: 39 y 40). Señalaron, además, que la esencia de la metáfora era entender y experimentar un tipo de cosa en términos de otra (Lakoff y Johnson, 2001: 41).

Una metáfora conceptual consiste en una proyección de un dominio fuente sobre un dominio meta. Por ejemplo, en la metáfora "la intransigencia es la inflexibilidad", el dominio fuente es la inflexibilidad y el dominio meta, la intransigencia. En este caso, la proyección de dominios se produce en expresiones metafóricas, tales como "El director es bastante rígido con la disciplina"2.

1 En su vertiente conceptual, el significado de "madre", por ejemplo, se define por hechos biológicos. En su vertiente cultural, en cambio, conlleva una serie de asociaciones relativamente complejas, como el brindar cariño y alimentar.

2 Según Cuenca y Hilferty (1999: 100), existe una diferencia entre las “expresiones metafóricas” y la "metáfora conceptual". Las primeras son las infinitas manifestaciones de las metáforas en los resultados de los actos de habla concretos. La metáfora, en cambio, es el esquema general que subyace a estas expresiones. Verbigracia, la metáfora "las teorías son edificios" se materializa en expresiones como "esta teoría carece de fundamentos" y "se derrumbó su teoría". 
Ahora bien, existe otro mecanismo de cambio lingüístico, la metonimia. Lakoff y Johnson la incluyen en el concepto de metáfora conceptual. La metonimia puede diferenciarse de la metáfora si se tiene en cuenta que no proyecta un dominio conceptual sobre otro, sino que opera dentro de un mismo dominio (Rodríguez Espiñeira, 2010: 33). Delbecque (2008: 37) señala que "se habla de metonimia cuando conceptualmente tomamos una cosa por otra, que percibimos como contigua [...] A diferencia de lo que sucede con la metáfora (donde entendemos un dominio en términos de otro), la metonimia es más bien un fenómeno referencial".

La metonimia (como mecanismo de cambio semántico) incluye la parte por el todo (sinécdoque: "había dos almas allí"), la causa por el efecto ("le hizo daño el sol"), el contenedor por el contenido ("tomar una copa"), el material por el objeto ("trajo los papeles"), el nombre de un productor por el producto ("tráeme maicena"), el nombre del autor por su obra ("este es un Goya"), el objeto utilizado por el usuario ("el segundo violín es muy bueno"), entre otras (Traugott y Dasher, 2002: 57).

Es importante aclarar que la metáfora y la metonimia son dos tipos de desplazamientos conceptuales diferentes, pero no incompatibles. Muchas expresiones de carácter "metonímico-inferencial"3 pueden dar como resultado el desplazamiento de una expresión desde un dominio origen hacia un dominio paralelo más abstracto (Elvira, 2009: 177) ${ }^{4}$.

\section{El embodiment}

Como se ha indicado en un trabajo anterior (Gonzalez, 2016: 30),

“en muchas ocasiones los hablantes no notan que están haciendo uso de metáforas debido a que estas están fuertemente arraigadas en el sistema conceptual humano y debido a que son motivadas por la experiencia cotidiana. Por ejemplo, hablar de la cantidad en términos de verticalidad ('más es arriba, menos es abajo') está basado en la experiencia de que, si vertemos un líquido en un recipiente, este sube a medida que aumenta su cantidad".

3 Que van adquiriendo nuevos valores contextuales progresivamente.

4 El ejemplo que presenta Elvira (2009: 178-182) es el del paso del deber con valor deóntico radical, que significaba obligación impuesta desde el exterior, al deber epistémico, que indica probabilidad: "En un principio, los verbos deber y poder se sitúan en un dominio regido por leyes físicas o circunstancias sociales que se ejercen sobre seres humanos, pero terminan situándose en un nuevo dominio de naturaleza mental y metalingüística. Se han producido, pues, sendas transferencias desde un dominio conceptual a otro, bastante parecidas a la traslación metafórica. Pero las transferencias de dominios son solo el efecto final y no la causa del cambio". A este respecto, consultar también Elvira (2006), Traugott y Dasher (2002: 29). 
Se puede observar en este caso que "las metáforas poseen a menudo un patrón de direccionalidad que va de lo concreto a lo abstracto" (Cuenca y Hilferty, 1999: 103). Más aún, "se dice lo concreto en el sentido de la interacción del propio cuerpo con el ambiente" (Gonzalez, 2016: 30), como ejemplifica Glenberg (2010: 587) aludiendo a Lakoff:

"Cuando las personas están tristes, literalmente se deprimen, se sientan o se desploman, mientras que cuando están felices, literalmente se yerguen y pueden literalmente saltar del gozo. Por lo tanto, la forma en la que pensamos sobre las emociones puede reflejar las interacciones literales de nuestros cuerpos con el ambiente"s.

Se ha señalado también en Gonzalez (2016: 8) que las gestalts experienciales fundamentan las metáforas conceptuales ha sido desarrollada por Johnson (1987), que habla de image schemata o embodied schemata, como señala Goschler (2005: 34-35).

"Un esquema de imagen es un patrón recurrente que emerge como estructuración del significado. Algunos de estos patrones son el movimiento en el espacio, la manipulación de objetos y las interacciones perceptivas. Forman parte de estos esquemas de imagen entre otros: camino-meta, las metáforas de contenedores y las metáforas orientacionales (arriba-abajo, frente-detrás)".

"En efecto, la mente humana con frecuencia saca partido de los dominios bien delimitados por la experiencia cotidiana para comprender otros, menos accesibles. Estos dominios concretos de los que se parte para comprender otros están constituidos por la experiencia corporal de cada persona. Cada uno se reconoce como un cuerpo que se desplaza en el espacio y está contenido en ciertos límites y extiende esa comprensión de sí mismo (en relación con las cosas, por supuesto) a la comprensión de las ideas más abstractas" (Gonzalez, 2016: 31). Esto es lo que se denomina embodiment, embodied mind o embodied cognition (Lakoff, 1987; Ziemke, 2003; Goschler, 2005; Soylu y otros, 2014).

Es necesario tener en cuenta que la teoría de la embodied cognition es relativamente nueva y no es aún homogénea. Como indican Soylu y otros (2014), "No es una teoría de la cognición unificada. Representa un conjunto de teorías apenas conectadas basadas en múltiples disciplinas con la noción común de un fundamento corporal para la cognición”.

¿En qué consiste este fundamento corporal de la cognición? Según Goschler (2005: 34), la noción de embodiment suele incluir no solo la idea de que razonamos a partir de las experiencias cotidianas de nuestro cuerpo, sino también la afirmación de que la razón misma depende especialmente de la estructura neurológica de nuestro cerebro.

5 "When people are sad, they literally slump, sit, or lie down, whereas when someone is joyous, they literally carry themselves erect and may literally jump for joy. Thus, the way we think about emotions may reflect the literal interactions of our bodies with the environment". 
Esta autora propone afinar el concepto de cuerpo y embodiment porque considera que se lo entiende de muy diversas maneras y que esta amplitud de interpretaciones lo banaliza. No cree que se deba llamar cuerpo a toda interacción del ser humano con el ambiente. Señala que es posible considerar todo comportamiento como una interacción entre el cuerpo y el ambiente, pero eso llevaría a trivializar la noción de cuerpo y, en ese sentido, sería mejor hablar simplemente de experiencia. Para evitar la trivialización del concepto de cuerpo y realizar una teoría no falseable propone afinar su definición (Goschler, 2005: 34).

Por otra parte, entender que la esencia del embodiment es el hecho de que la cognición está unida de modo inseparable a los procesos cerebrales le parece a la autora una ampliación excesiva del alcance del término, pues todos los procesos cognitivos pasan por el cerebro, todos ellos son corporalizados. No habría especificidad del término embodiment.

Goschler (2005: 35) va incluso más allá con su crítica: argumenta contra el hecho de que se busque una base corporal para la totalidad de las proyecciones metafóricas. Según la autora, las metáforas conceptuales no tienen que estar basadas necesariamente en la experiencia corporal. Esto no significa que los conceptos de "metáfora conceptual" y embodiment no se hallen fuertemente enlazados, pues es claro que poseen una relación que se manifiesta en las que propiamente se pueden llamar "metáforas del cuerpo", en especial en una clase dentro de este tipo de metáforas, como se verá a continuación.

Las metáforas del cuerpo incluyen tres subclases. La primera es el uso de expresiones pertenecientes al dominio del cuerpo (partes u órganos del cuerpo y características físicas como la fuerza y la debilidad o la enfermedad y la salud) para describir otras cosas (la comunicación). Algunos ejemplos de esta clase de metáforas del cuerpo son: "las máquinas y computadoras son un cuerpo", "las comunidades (equipos, naciones, etc.) son cuerpos" y "la comunicación es un cuerpo" (Goschler, 2005: 37).

La segunda clase es la que hace lo contrario: utiliza otros dominios (el de las plantas) para describir partes-órganos del cuerpo humano. Un ejemplo de este tipo de metáforas es "los ojos son cámaras" (Goschler, 2005: 42). Un tercer grupo es el que se caracteriza por el uso de partes del cuerpo humano o estados físicos para hacer referencia a sentimientos o emociones ("me hirvió la sangre"). En esta clase de metáforas es difícil cuál es el dominio fuente y cuál es el dominio meta.

Como señala Goschler (2005: 36), los estudiosos que arguyen que las metáforas del cuerpo son una prueba de la tesis del embodiment se centran en la primera clase de metáforas señalada (aquella en la que el cuerpo es dominio fuente). No obstante, como puede observarse en las otras clases de metáforas del cuerpo, las proyecciones metafóricas pueden darse en distintas direcciones (de lo concreto a lo abstracto y viceversa).

Glenberg (2010: 586) propone la siguiente definición de embodiment: "a pesar de que existen diferentes versiones de la teoría del embodiment, la mayoría de ellas toma como 
punto de partida el hecho de que los procesos psicológicos se encuentran influenciados por el cuerpo, lo cual incluye su morfología, sus sistemas sensoriales y sus sistemas motores”.

Ziemke particulariza un poco esta delimitación: "En orden a evitar una pérdida de significado de la palabra embodiment haciéndola totalmente polisémica, propongo un uso del término en lingüística cognitiva solo en el primer sentido: embodiment significa que parte de nuestro sistema conceptual y, por lo tanto, algunos aspectos de nuestro lenguaje están estructurados por las características de nuestro cuerpo y su funcionamiento en la vida cotidiana. Esta definición es aún borrosa y puede incluir diferentes clases de embodiment" ${ }^{7}$ (ápud Goschler, 2005: 35).

Esta noción de embodiment no deja de lado la idea del embodiment social. Se ha repetido mucho la afirmación de que los códigos culturales influyen sobre el cuerpo, pero también se da el camino inverso. Existen simulaciones corporales que son la base de los símbolos negociados en los contextos socioculturales y que se repiten socialmente hasta cristalizarse en juegos de lenguaje, rituales, entre otros (Soylu y otros, 2014: 4) ${ }^{8}$. Glenberg (2010: 589) ejemplifica esto con las neuronas espejo: "el reclamo del embodiment para el lenguaje es que las oraciones son entendidas simulando su contenido a través del uso de sistemas neurológicos usados ordinariamente para la percepción, la acción y la emoción"9. Las neuronas espejo son aquellas neuronas de áreas motoras que se activan cuando se ve a otra persona correr e incluso cuando se escuchan verbos como correr.

Otro ejemplo de la estrecha relación existente entre el cuerpo y el contexto sociocultural es el de la influencia de los estados corporales sobre la interacción social. Glenberg (2010: 591) cita a este respecto un experimento de Williams y Bargh (2008). Testearon la hipótesis de que el calor físico es extendido metafóricamente al dimensiones de "calor social" como la amistad y la solidaridad.

Los participantes del experimento debían sostener unos momentos una taza de café caliente o frío. Mientras, se les pedía que eligieran un regalo para ellos mismos o para un amigo. El $54 \%$ de los que sostuvieron la taza caliente eligieron el presente para un amigo, mientras que de los que sostuvieron el objeto frío, sólo el 25\% eligió el regalo para un amigo. Por consiguiente, cambiando el cuerpo (en este caso, una característica de su funcionamiento) cambian el comportamiento social de las personas.

6 "Although there are different versions of embodiment theory, for the most part, they take as a starting point that psychological processes are influenced by the body, including body morphology, sensory systems, and motor systems".

7 "To avoid a loss of meaning of "embodiment" by making it totally polysemous I argue for a use of the term in Cognitive Linguistics in only the first sense: Embodiment means that parts of our conceptual system and therefore some aspects of our language are structured by the features of our bodies and the functioning of our bodies in everyday life. This definition is still fuzzy and can include different sorts of embodiment".

8 Soylu y otros (2014) estudian cómo los gestos en el contexto de la enseñanza-aprendizaje muestran el embodiment (contar con los dedos), la mímica y la imitación.

9 "The embodiment claim for language is that sentences are understood by simulating sentence content using neural systems ordinarily used for perception, action, and emotion". 


\section{Conclusiones y corolarios}

El abordaje cognitivo-funcional de los cambios en la lengua aporta un enriquecimiento de los estudios sobre este tema, pues toma en consideración los procesos cognitivos del ser humano y considera la lengua como un instrumento de comunicación. En este trabajo se señaló la importancia que poseen dos factores en una concepción cognitivista del lenguaje. El primero de ellos es la metáfora conceptual, noción que se refiere a que constantemente proyectamos ciertos dominios conceptuales sobre otros.

El segundo, visto en muchos casos como la base del primero, es el embodiment. Si bien no existe una teoría unificada de la cognición corporizada, puede decirse que, en general, el concepto de embodiment hace referencia a que la cognición está influida por la morfología del cuerpo, sus estados y su experiencia en relación con el entorno.

En otras palabras, las partes del cuerpo, los cambios en su funcionamiento y aquellos derivados de su interacción con otros cuerpos conducen el pensamiento humano hacia una determinada dirección u otra y actúan consciente o inconscientemente en la vida cotidiana (como lo indica esta misma metáfora corporal que acabamos de usar, que habla de conducir el pensamiento, darle movimiento).

\section{Fuentes consultadas}

Company, C. (2003). “¿Qué es un cambio lingüístico?”, pp. 13-33. En Colombo, F. y Soler, M. (coords.). Cambio lingüístico y normatividad. México D. F.: UNAM.

Cuenca, M. y Hilferty, J. (1999). Introducción a la lingüistica cognitiva. Barcelona: Ariel.

Delbecque, N. (2008). "Semántica cognitiva y categorización lingüística", pp. 19-56. En Rodríguez Espiñeira, M. y Pena Seijas, J. (eds.). Categorización lingüística y límites intercategoriales. Santiago de Compostela: Servizo de Publicacións e Intercambio Científico.

Echeverría Arriagada, C. (2012). "Hacia el estudio crítico de la variación y el cambio lingüísticos”. Bajo Palabra. Vol. 7, núm. 2, pp. 565-573.

\section{Elvira, J.}

_(2009). Evolución lingüística y cambio sintáctico. Berna: Peter Lang.

_(2006). "Sobre el desarrollo del valor epistémico del verbo poder”, pp. 641-654. En Girón, J. y De Bustos, J. (coords.). Actas del VI Congreso Internacional de Historia de la Lengua española. Vol. 1. Madrid, 29 de septiembre-3 de octubre de 2003.

Frank-Job, B. (2010). “Cambio lingüístico y variación lingüística: Sobre el significado de las tradiciones discursivas para la investigación del cambio lingüístico”. En Gallegos-Shibya, A. y Iturrioz Leza, J. (eds.). Tradiciones discursivas. Guadalajara: Universidad de Guadalajara. 
Fundéu BBVA(2014). “Selfi, palabra del año para la Fundéu BBVA”. Extraída el 24/X/2017 desde http://www.fundeu.es/recomendacion/selfi-palabra-del-ano-para-la-fundeu-bbva/

Gallegos Shibya, A. (2003). Nominalización y registro técnico. Algunas relaciones entre morfopragmática, tradiciones discursivas y desarrollo de la lengua en español [tesis doctoral]. Friburgo: Universidad de Friburgo. Extraída el 24/X/2017 desde http://www.freidok.uni-freiburg.de/volltexte/2622/pdf/Gallegos_Shibya_Dissertation.pdf

Geeraerts, D. (ed.) (2006). Cognitive Linguistics: Basic Readings. Berlin: Mouton de Gruyter.

Gibbs, R. (1992). "When Is Metaphor? The Idea of Understanding in Theories of Metaphor”. Poetics Today. Vol. 4, núm. 13, pp. 575-606.

Glenberg, A. (2010). "Embodiment as a Unifying Perspective for Psychology”. Cogn Sci, núm. 1, pp. 586-596.

\section{Gonzalez, D.}

_(2016). La lingüística cognitiva y su teorización sobre la metáfora conceptual. Principales visiones históricas sobre el fenómeno y caracterización desde los principios cognitivofuncionales. Saarbrücken: Editorial Académica Española. Extraída el 24/X/2017 desde https://es.scribd.com/document/307026404/Libro-Metafora-Final

_(2013). "María José Rodríguez Espiñeira, y Jesús y Pena Seijas (eds.) (2008), Categorización lingüística y límites intercategoriales, Santiago de Compostela, Servizo de Publicacións e Intercambio Científico-Universidad de Santiago de Compostela" [reseña de libro]. Signos Linguísticos. Vol IX., núm. 17, pp. 125-131. Extraída el 24/X/2017 desde http://signoslinguisticos.izt.uam.mx/index.php/SLING/article/view/125/109

Goschler, J. (2005). “Embodiment and Body Metaphors". Extraída el 24/X/2017 desde http://www.metaphorik.de/09/goschler.htm

Kabatek, J. (2005). “Tradiciones discursivas y cambio lingüístico”. Lexis. Vol. 29, núm. 2, pp. 151-177.

Lakoff, G. (1987). Women, Fire and Dangerous Things. What Categories reveal about the Mind. Chicago: University of Chicago Press.

Lakoff, G. y Johnson, M. (2001). Metáforas de la vida cotidiana. Madrid: Cátedra.

Rodríguez Espiñeira, M. (coord.) (2010). Adjetivos en discurso. Emociones, certezas, posibilidades y evidencias. Santiago de Compostela: USC.

Soylu, F. y otros (2014). “The Thinking Hand: Embodiment of Tool Use, Social Cognition and Metaphorical Thinking and Implications for Learning Design". Extraída el 24/X/2017 desde http://ccl.northwestern.edu/2014/Soylu-2014-ThinkingHand.pdf 
Traugott E. y Dasher, R. (2002). Regularity in Semantic Change. Nueva York: Cambridge University Press.

Ziemke, T. (2003). "What's that thing called Embodiment?”, pp. 134-1139. En Alterman, R. y Kirsh, D. (eds.). Proceedings of the 25th Annual Conference of the Cognitive Science Society. Nueva Jersey: Lawrence Erlbaum. Extraída el 24/X/2017 desde https://pdfs.semanticscholar.org/8cd4/1ddebb70d2652c52ed5c6ba126179302de69.pdf 